\title{
Fisioterapia e GOVID-19: das repercussões sistêmicas aos desafios para oferta de reabilitação
}

Helena Fraga-Maia ${ }^{1}$,Elen Beatriz Pinto ${ }^{2}$, Ítalo Ricardo Santos Aleluia ${ }^{3}$, Luciana Limoeiro Ricarte Cavalcante ${ }^{4}$, Rhaine Borges Santos Pedreira, Thelso de Jesus Silva ${ }^{6}$, Thiago Santos de Souza ${ }^{7}$, Juliana Martins Pinto ${ }^{8}$, Elzo Pereira Pinto Junior ${ }^{9}$

1 Fisioterapeuta, mestre em Saúde Comunitária e doutora em Saúde Pública pelo Instituto de Saúde Coletiva (ISC) da Universidade Federal da Bahia (UFBA), professora titular da Universidade do Estado da Bahia (UNEB) e pesquisadora convidada do Estudo Longitudinal de Saúde do Adulto (ELSA-Brasil).

2 Fisioterapeuta, mestre em Medicina e Saúde e doutora em Ciências da Saúde pela Universidade Federal da Bahia (UFBA). Pesquisadora, professora titular e orientadora nos programas de mestrado e doutorado da Escola Bahiana de Medicina e Saúde Pública (EBMSP) e professora adjunta da Universidade do Estado da Bahia (UNEB).

3 Fisioterapeuta, mestre em Saúde Comunitária e doutor em Saúde Pública pelo Instituto de Saúde Coletiva (ISC) da Universidade Federal da Bahia (UFBA). Docente da Universidade Federal do Oeste da Bahia (UFOB).

4 Fisioterapeuta, residente em Planejamento e Gestão em Saúde Coletiva pelo Instituto de Saúde Coletiva (ISC) da Universidade Federal da Bahia (UFBA).

5 Fisioterapeuta pela Universidade Estadual do Sudoeste da Bahia (UESB). Mestre e doutoranda em Ciências da Saúde pelo Programa de Pós-Graduação em Enfermagem e Saúde (PPGES) da UESB.

6 Fisioterapeuta, mestre em Medicina e Saúde pela Universidade Federal da Bahia (UFBA). Especialista em Fisioterapia respiratória e Terapia intensiva pela Associação Brasileira de Fisioterapia Cardiorrespiratória e Fisioterapia em Terapia Intensiva (Assobrafir) e Conselho Federal de Fisioterapia e Terapia Ocupacional (Coffito). Coordenador do serviço de fisioterapia do Hospital São Rafael, em Salvador (BA).

7 Fisioterapeuta, mestre em Saúde Comunitária e Doutorando em Saúde Pública pelo Instituto de Saúde Coletiva (ISC) da Universidade Federal da Bahia (UFBA). Docente da Escola Bahiana de Medicina e Saúde Pública (EBMSP).

8 Fisioterapeuta, especialista em Gerontologia pela Escola Paulista de Medicina (EPM) da Universidade Federal de São Paulo (Unifesp) e Associação Brasileira de Geriatria e Gerontologia (SBGG). Doutora em Gerontologia pela Universidade Estadual de Campinas (Unicamp). Pós-doutorado em Epidemiologia do Envelhecimento pela Universidade Federal do Rio Grande do Norte (UFRN). Docente na Universidade Federal do Triângulo Mineiro (UFTM).

9 Fisioterapeuta pela Universidade Estadual do Sudoeste da Bahia (UESB). Mestre em Saúde Coletiva Universidade Estadual do Ceará (UECE). Doutor em Saúde Pública pelo Instituto de Saúde Coletiva (ISC) da Universidade Federal da Bahia (UFBA). Pós-doutorando em Epidemiologia no Centro de Integração de Dados e Conhecimentos para a Saúde (Cidacs) da Fundação Oswaldo Cruz (Fiocruz) da Bahia.

FRAGA-MAIA, H.; PINTO, E. B.; ALELUIA, Í. R. S.; CAVALCANTE, L. L. R.; PEDREIRA, R. B. S.; SILVA, T. de J.; SOUZA, T. S. de; PINTO, J. M.; PINTO JUNIOR, E. P. Fisioterapia e COVID-19: das repercussões sistêmicas aos desafios para oferta de reabilitação. In: BARRAL-NETTO, M.; BARRETO, M. L.; PINTO JUNIOR, E. P.; ARAGÃO, E. (org.). Construção de conhecimento no curso da pandemia de COVID-19: aspectos biomédicos, clínico-assistenciais, epidemiológicos e sociais. Salvador: Edufba, 2020. v. 1.

DOI: https://doi.org/10.9771/9786556300443.011. 


\section{Introdução}

A pandemia de COVID-19 tem produzido inúmeros desafios para a organização dos serviços de saúde. O grande contingente de pacientes que foram internados por causa do novo coronavírus sobrecarregou os hospitais, especialmente as Unidades de Terapia Intensiva (UTI), haja vista a necessidade de suporte ventilatório nos casos mais graves. $\mathrm{O}$ manejo desses pacientes demanda muitas intervenções do fisioterapeuta, profissional responsável por prevenir e tratar alterações funcionais nos sistemas respiratório, musculoesquelético e neurológico. (IANNACCONE et al., 2020) Adicionalmente, as repercussões da COVID-19 decorrentes de longos períodos de hospitalização também são alvos das intervenções fisioterapêuticas, tanto na atenção ambulatorial quanto na Atenção Primária à Saúde (APS). Desse modo, a assistência fisioterapêutica no pós-alta é imprescindível para garantir funcionalidade, autonomia e qualidade de vida. (KALIRATHINAM; GURUCHANDRAN; SUBRAMANI, 2020; SHEERY, 2020)

Este capítulo tem como objetivo discutir os impactos funcionais decorrentes das repercussões clínicas secundárias à infecção pelo SARS-CoV-2, assim como as abordagens e os desafios para a oferta de atendimento fisioterapêutico no âmbito do Sistema Único de Saúde (SUS). 


\section{Repercussões sistêmicas da COVID-19 e abordagens da fisioterapia em pacientes internados nos serviços especializados}

O acometimento do sistema respiratório é a manifestação clínica predominante da COVID-19, apresentando-se como doença leve semelhante à gripe, podendo evoluir para a síndrome do desconforto respiratório agudo potencialmente letal ou pneumonia fulminante. (STAWICKI et al., 2020) Aproximadamente 14\% dos pacientes com COVID-19 desenvolvem doença respiratória, e estes geralmente apresentam febre, tosse seca, dispneia e infiltrados pulmonares bilaterais nas imagens do tórax. (WU; MCGOOGAN, 2020) As complicações da COVID-19 incluem a Síndrome Respiratória Aguda (SDRA), insuficiência respiratória, lesão hepática, lesão miocárdica aguda, lesão renal aguda, choque séptico e até mesmo falência de múltiplos órgãos. (SHI et al., 2020) Fatores de risco cardiovasculares preexistentes aumentam a vulnerabilidade à COVID-19 e a idade avançada, as comorbidades, tais como hipertensão, diabetes mellitus e doença pulmonar crônica têm sido associadas com resultados desfavoráveis. (MADJID et al., 2020)

A abordagem fisioterapêutica dos pacientes graves em UTI envolve, principalmente, o manejo do suporte ventilatório com oferta de oxigênio. Entretanto, os procedimentos para esse manejo em pacientes com COVID-19 diferem dos usuais, devido à necessidade de reduzir ou eliminar a produção de aerossois que contaminariam o ambiente, profissionais e outros pacientes. (BATTAGLINI et al., 2020) Por isso, o uso de cânula nasal de alto fluxo, máscara de Venturi e Ventilação Mecânica Não Invasiva (VMNI) não é recomendado. Foi apontado que a VMNI pode promover atraso na intubação, associando-se a maior mortalidade. (ALHAZZANI et al., 2020) As recomendações para o manejo desses 
pacientes são cateter de oxigênio, máscara facial com reservatório, intubação orotraqueal e ventilação mecânica. Além disso, recomenda-se que o ambiente tenha um sistema de pressão negativa para minimizar a contaminação pelo vírus. (BATTAGLINI et al., 2020) No manejo da ventilação mecânica há recomendação de uma estratégia ventilatória protetora, caracterizada por baixos volumes, controle da pressão platô e monitoramento da driving pressure - diferença entre platô de pressão inspiratória e a Pressão Expiratória Final Positiva (PEEP). O uso de volume e pressão alveolar elevados foram associados ao aumento na mortalidade nos pacientes com SDRA. (FAN et al., 2017)

A atuação da equipe de fisioterapia na UTI deve considerar a instabilidade hemodinâmica e a baixa tolerância ao exercício. Destaca-se a importância da monitorização da troca gasosa e mecânica respiratória, da avaliação seriada dos parâmetros gasométricos, complacência estática, resistência e pressão de distensão (driving pressure). A análise gráfica da ventilação pode auxiliar nas tentativas de melhora de hipoxemia com menor risco de lesão pulmonar. (LAZZERI et al., 2020) A avaliação da aeração através do ultrassom pulmonar tem sido relevante para os pacientes com COVID-19, por ser não invasiva e segura. (OLIVEIRA et al., 2020)

A fisioterapia colabora para a prevenção de Pneumonia Associada à Ventilação (PAV), com manutenção da cabeceira do leito elevada e mensuração da pressão do cuff como rotina para evitar despressurização, contaminação das vias aéreas inferiores e ventilação inadequada. É relevante a atenção do fisioterapeuta quanto ao posicionamento do paciente, não só durante os procedimentos de posição prona, que será feito por equipe treinada e protocolo específico (GUÉRIN et al., 2013), mas na análise contínua da resposta da oxigenação à cada novo posicionamento.

No processo de desmame da Ventilação Mecânica (VM), recomenda-se avaliação da estabilidade clínica e hemodinâmica, 
redução de drogas vasoativas, melhora da hipoxemia e PEEP reduzida. (SCHMIDT et al., 2017) É necessário um bom nível de consciência para realização do Teste de Respiração Espontânea (TRE), que será o principal marcador para a tomada de decisão de descontinuação da VM. (BOLES et al., 2007) O modo de Ventilação com Pressão de Suporte (PSV) é recomendado, inclusive para a realização do TRE. (RIGHETTI et al., 2020) Após o TRE bem-sucedido, o fisioterapeuta deverá considerar o reflexo de tosse como indicador da capacidade de proteção da via aérea e não realizar teste de escape do cuff antes da extubação, devido à disseminação de gotículas. Recomenda-se que os pacientes sejam extubados, preferencialmente, em ambiente com pressão negativa ou em isolamento respiratório, com atenção às precauções de isolamento, incluindo a manutenção de um filtro trocador de calor e umidade conhecido como Heat and moisture Exchanger Filter (HMEF) e o sistema fechado de aspiração conectado ao tubo no momento da desinsuflação do cuff. Recomenda-se evitar manipulação vigorosa e tosse.

A realização de mobilização precoce é determinante no resultado funcional a longo prazo, com influência na dependência funcional e necessidades de cuidados. (HODGSON et al., 2014) Além disso, a imobilidade prolongada do paciente promove risco elevado de tromboembolismo venoso e as condutas fisioterapêuticas possibilitam a prevenção desses eventos. Todavia, é preciso considerar o equilíbrio entre a mobilização precoce e o gasto energético excessivo nos pacientes com hipermetabolismo (ou febre). Pacientes sedados e curarizados, mesmo com consumo metabólico reduzido, desenvolvem risco de sequelas neuromusculares, descondicionamento, contraturas musculares e lesões por pressão. Em casos de SDRA associada à COVID-19, dados de mortalidade e morbidade em longo prazo não estão disponíveis atualmente e, até o presente momento, não foi quantificado na literatura se as alterações do tecido pulmonar, como opacidades em 
vidro fosco, consolidação, espessamento vascular e outros, podem ser permanentes, quais serão os danos físicos para os pacientes no longo prazo, ou se esses achados serão reversíveis. Após alta da UTI, os pacientes devem continuar a reabilitação individualizada supervisionada.

A ventilação mecânica prolongada e a internação por longos períodos na UTI promovem sequelas, citadas na literatura como "fraqueza adquirida na UTI", "polineuropatia do doente crítico" e outras. (STAM; STUCKI; BICKENBACH, 2020) Pacientes com o vírus tendem a necessitar de VM por mais tempo do que a média e, assim, estarão propensos às alterações semelhantes aos pacientes com SDRA, com alto nível de descondicionamento e desfechos desfavoráveis em curto e longo prazo. (NEEDHAM et al., 2017) Observou-se capacidade funcional comprometida após alta, através de teste de caminhada de 6 minutos (TC6'), com apenas $49 \%$ dos pacientes chegando à distância esperada para a faixa etária e 75\% chegando a essa marca somente um ano após a alta. (HERRIDGE et al., 2011)

Estimativas avaliam que 5 a 10\% dos pacientes hospitalizados terão indicação para reabilitação extra-hospitalar pós-COVID-19, já que uma fração dos pacientes não ventilados também precisará ser reabilitada pelas consequências da internação. (DOCHERTY et al., 2020) Sintomas como fadiga e dispneia ocorrem com frequência, cerca de até 30 dias após a alta hospitalar e são acompanhados por redução da qualidade de vida em $40 \%$ dos pacientes. (FRIJA-MASSON et al., 2020)

Sabe-se que a COVID-19 afeta principalmente a via respiratória e o sistema cardiovascular, no entanto, o envolvimento neurológico não é incomum e pode resultar em complicações, principalmente em pacientes gravemente doentes. A doença neurológica pode ser consequência de uma doença generalizada, da insuficiência cardiorrespiratória, das anormalidades metabólicas desencadeadas pela infecção, da invasão direta do vírus ou de uma 
resposta autoimune ao mesmo. Os mecanismos neuropatológicos subjacentes aos danos ao Sistema Nervoso Central (SNC) podem estar relacionados a uma lesão cerebral hipóxica resultante de pneumonia grave, levando a dano cerebral. Os fatores contribuintes incluem vasodilatação periférica, hipercarbia, hipóxia e metabolismo anaeróbio com acúmulo de compostos tóxicos, que pode resultar em edema cerebral e em dano neurológico. A lesão imunomediada se deve principalmente às tempestades de citocinas, com níveis aumentados de citocinas inflamatórias e ativação de linfócitos T, macrófagos e células endoteliais. A liberação adicional de interleucinas causa vazamento vascular, cascata de coagulação, coagulação intravascular disseminada e lesão de órgão-alvo. (AHMAD; RATHORE, 2020)

Os estudos trazem também que a infecção por COVID-19 pode prejudicar o sistema nervoso através da invasão direta do tecido neural. (WHITTAKER; ANSON; HARKY, 2020) Dessa maneira, o SARS-CoV-2 pode afetar o SNC principalmente através da via hematogênica e de rotas neuronais retrógradas. O SARS-CoV-2 pode invadir primeiro terminais nervosos periféricos e infectar o SNC retrogradamente, haja vista que a anosmia e disgeusia foram relatados como prevalentes entre pacientes com COVID19, mesmo sem haver sintomas nas vias respiratórias superiores, o que sugere que o vírus pode afetar a função olfativa via invasão direta do neuroepitélio olfatório. (WANG et al., 2020)

As manifestações neurológicas na COVID-19 estão distribuídas principalmente em três categorias (PINZON et al., 2020), a saber:

1. doenças neurológicas comórbidas com COVID-19 faz os pacientes mais suscetíveis à COVID-19 devido à exposição frequente em instalações médicas e a um estado de saúde abaixo do ideal; 
2. manifestações neurológicas inespecíficas que podem ser causadas parcialmente por respostas ao comportamento neuroinvasivo da infecção; e

3. sintomas neurológicos específicos e doenças causadas por envolvimento neurológico em COVID-19.

As manifestações neurológicas não específicas podem ocorrer na fase precoce da infecção e podem ser o principal e único sintoma em pacientes internados por COVID-19. Sintomas como a dor de cabeça, mialgia, fadiga, náusea/vômito, confusão mental, anorexia, tontura, mal-estar e dispneia foram relatados como sintomas neurológicos inespecíficos. A cefaleia é um dos sintomas mais comum, com surgimento precoce e agravamento no acompanhamento. (PINZON et al., 2020) Tipicamente, a dor de cabeça é considerada leve e a frequência com que ocorre, devido a uma meningite viral, permanece incerta. Entre as manifestações clínicas mais frequentes estão a fadiga, dispneia, anorexia e mal-estar. Se a dispneia é um fator considerado como sintoma neurológico ainda é uma questão de debate, considerando a relação da dispneia, com a necessária respiração ativa e consciente, para manter um ritmo respiratório normal. Dada a alta similaridade entre coronavírus e o fato de que outros coronavírus por exemplo, o SARS-CoV e o MERS-CoV poderiam invadir o sistema nervoso, o vírus SARS-CoV-2 pode ter um papel na dispneia, afetando o centro respiratório, especialmente naqueles pacientes com rápida progressão da doença, mas sem sintomas respiratórios graves. (MONTALVAN et al., 2020)

As manifestações neurológicas são mais prevalentes em pessoas gravemente doentes, e podem levar à morte se não forem tratadas adequadamente. (BERGER, 2020) Os sintomas podem estar relacionados ao SNC, como níveis alterados de consciência, encefalite, doença cerebrovascular aguda, sinais do trato corticoespinhal, 
ataxia e convulsão. Dentre os sintomas relacionados ao Sistema Nervoso Periférico (SNP), são encontrados o comprometimento do paladar (ageusia), do olfato (anosmia), deficiência visual, dor nos nervos, mialgia e miosite. (LEONARDI; PADOVANI; MCARTHUR, 2020) Em muitos casos, a miopatia apresentada é frequentemente atribuída à neuropatia do doente crítico, embora a invasão viral não possa ser totalmente excluída. Curiosamente, disfunção olfativa e distúrbios gustativos foram observados em 30 a $80 \%$ dos pacientes com quadro leve de COVID-19. (BERGER, 2020)

Como em muitas doenças virais, a infecção por SARS-CoV-2 pode desencadear uma resposta autoimune afetando o SNC e o SNP. A neuroinflamação parece estar relacionada com a COVID-19, incluindo a síndrome de Guillain-Barré, síndrome de Miller Fisher, mielite, meningite e encefalite. O mecanismo para a neuroinflamação nos pacientes com COVID-19 não é claro, suspeita-se que tanto a tempestade de citocinas secundária à infecção sistemática, quanto a hiperativação do sistema imunológico podem ter um papel nesse processo. (PINZON et al., 2020)

Não está claro como a infecção por COVID-19 modifica o curso clínico de doença neurológica preexistente. Em muitas ocasiões, os mecanismos imunológicos que desencadeiam a tempestade de citocinas, típicas de síndromes graves de SARS, tem um papel relevante na iniciação de várias doenças neurodegenerativas, bem como em sua progressão. (SERRANO-CASTRO et al., 2020) A neuroinflamação crônica decorrente dos altos níveis de citocinas/quimiocinas têm sido associadas à fisiopatologia de algumas doenças neurodegenerativas como esclerose múltipla, doença de Parkinson, Doença de Alzheimer, doença de Huntington ou esclerose lateral amiotrófica. (FERINI-STRAMBI; SALSONE, 2020) Ademais, essa atividade neuroinflamatória pode ter também repercussão nas doenças psiquiátricas, caso a infecção com SARS-CoV-2 siga um curso semelhante ao do SARS-CoV ou MERS-CoV, a maioria dos 
pacientes deve se recuperar. O SARS-CoV-2 pode causar delirium em uma proporção significativa de pacientes na fase aguda. A função cognitiva e neuropsicológica deve ser monitorada de perto em sobreviventes de COVID-19 (SERRANO-CASTRO et al., 2020) e os profissionais devem estar cientes da possibilidade de depressão, ansiedade, fadiga, transtorno de estresse pós-traumático e síndromes neuropsiquiátricas mais raras em longo prazo. (FERINI-STRAMBI; SALSONE, 2020)

Os efeitos do SARS-CoV-2 podem variar entre as doenças neurodegenerativas e doenças neuroimunes, a depender de aspectos como a vulnerabilidade para a infecção, modificação do curso clínico da doença, progressão da doença, gatilho para neurodegeneração futura e estratégias inovadoras para apoiar os profissionais no manejo da doença. Devido ao alto risco de infecção cruzada os pacientes com COVID-19, durante a pandemia, podem não fazer exames de neuroimagem avançada, testes eletrofisiológicos e exames patológicos, o que pode limitar a evidência de envolvimento neurológico. Entretanto, as pesquisas apontam ser altamente provável que alguns desses pacientes, particularmente aqueles que sofrem de uma doença grave, tenham envolvimento do SNC e manifestações neurológicas. O registro preciso e direcionado dos sinais e sintomas neurológicos, investigações clínicas e eletrofisiológicas detalhadas dos pacientes - particularmente aqueles com uma mudança do status mental -, tentativas de isolar SARS-CoV-2 e autópsias das vítimas de COVID-19, podem esclarecer os papéis desempenhados por esse vírus em causar as manifestações neurológicas. (ASADI-POOYA; SIMANI, 2020; GHANNAM et al., 2020; PINZON et al., 2020)

Além das manifestações respiratórias e neurológicas, os sintomas musculoesqueléticos têm sido reportados em vários trabalhos com incidência variando de um quarto à metade dos pacientes sintomáticos. (HEYDARI et al., 2020) Todavia, em um 
estudo multicêntrico envolvendo 417 pacientes com quadro clínico moderado ou grave, a ocorrência de mialgia foi de $59 \%$. (LECHIEN et al., 2020) Mialgias extensas e disfunções musculares já haviam sido descritas como secundárias à infecção por SARS-CoV (CHERRY; KROGSTAD, 2004; FANG, 2003) em pacientes que evoluíram com quadros graves e que, após dois a três meses da alta hospitalar, apresentaram redução de $32 \%$ na força de preensão palmar e de $13 \%$ na distância percorrida no teste de 6 minutos. (LAU et al., 2005)

São também frequentes as queixas de fadiga, cuja incidência varia entre $25,6 \%$ a $50,0 \%$, de artralgia $(31,0 \%)$, de dor nas costas $(10,3 \%)$ e de fraqueza muscular (25\% a 50\%). (CIPOLLARO et al., 2020) De modo geral, essas queixas são reportadas pelos pacientes desde o início dos sintomas e com maior ocorrência entre os que desenvolvem quadros clínicos moderados ou graves, com internamento em unidades de terapia intensiva. (CIPOLLARO et al., 2020; HEYDARI et al., 2020) Estima-se que dentre os pacientes acometidos pela COVID-19 que necessitaram de hospitalização e admissão em UTI, a média de permanência nesses ambientes foi de aproximadamente 10 e 14 dias, respectivamente. (TURCOTTE et al., 2020) Nesse sentido, resultados de uma revisão sistemática apontaram que cerca de $40 \%$ dos pacientes admitidos em UTI apresentaram um quadro de fraqueza adquirida devido ao tempo de restrição de movimento no leito. (APPLETON; KINSELLA; QUASIM, 2015)

O uso de corticosteroides, que são prescritos para limitar a inflamação nas fases iniciais da infecção, pode induzir diretamente a atrofia e fraqueza muscular e o descondicionamento físico, decorrente do período de internação hospitalar e de convalescência, também concorre para tais desfechos. (LAU et al., 2005) Vale ressaltar que a desmielinização neuronal, que já havia sido descrita em pacientes com SARS-CoV pode contribuir com fraqueza muscular. (DING et al., 2003) Foi relatado ainda 
rabdomiólise em pacientes infectados pelo SARS-CoV-2, todavia não está claro se essa complicação se deve ao dano muscular induzido pelo vírus ou pela reação autoimune secundária à doença. (HUSAIN et al., 2020)

Calcificação heterotópica e dor prolongada também foram referidas em pacientes com diagnóstico de SDRA submetidos à ventilação mecânica. (TANSEY et al., 2007) A ocorrência de osteonecrose e de redução de densidade óssea foram complicações observadas no sistema musculoesquelético em pacientes com SARS, apesar desses achados não terem sido relacionados com a doença em si, mas sim com as altas doses de corticoides utilizadas no seu manejo. (GRIFFITH, 2011) Esse conjunto de manifestações musculoesqueléticas é consequência, em grande parte, de longos períodos de hospitalização e, portanto, passíveis de serem encontrados em pacientes com COVID-19 que demandem internações de longa duração.

Por fim, outros aspectos também devem ser considerados quando se trata de pacientes hospitalizados por COVID-19, como déficits nutricionais relacionados à dificuldade na ingestão de alimentos, que em alguns casos ocorrem por sondas, ou ainda decorrentes de náusea, vômito ou diarreia, sintomas que vêm sendo relatados por pacientes com a doença. Todos esses fatores aumentam o risco para o declínio do sistema musculoesquelético. (BIASE et al., 2020)

\section{Impacto da COVID-19 na funcionalidade e abordagem fisioterapêutica}

O impacto da COVID-19 nas estruturas e funções dos diversos sistemas corporais vem sendo amplamente avaliado e debatido, entretanto, as informações sobre o quanto essas disfunções refletem no desempenho em Atividades de Vida Diária (AVD) e na participação 
social são escassas. Sabe-se que essas limitações e restrições provavelmente serão somadas às atuais demandas por assistência fisioterapêutica, configurando um cenário pós-pandemia preocupante e desafiador para a organização dos sistemas de saúde e para a sociedade. (DEAN et al., 2020)

As limitações nas atividades e restrição da participação social secundárias às disfunções pós-COVID-19 podem ser compreendidas a partir de duas perspectivas. Por um lado, a doença cursa com sintomas que podem persistir após a recuperação da infecção, bem como sequelas motoras, respiratórias, cardiocirculatórias, neurológicas, cognitivas e emocionais. Tais aspectos acompanham o indivíduo após a alta hospitalar, limitando seu desempenho em AVD e comprometendo sua independência funcional, de modo que este precise de ajuda parcial ou total para realizar o autocuidado. Por outro lado, a despeito dos sintomas e sequelas causados pela doença infecciosa, soma-se ao cenário de demandas pós-COVID-19 os efeitos adversos da exposição prolongada à restrição de movimentos no leito hospitalar, à ventilação mecânica, aos efeitos colaterais de medicamentos e outras terapias. Nesse contexto, há aumento da incidência de confusão mental aguda, sarcopenia, incontinência urinária e infecção, disfagia, e outras complicações, relacionadas ao ambiente hospitalar e às condutas adotadas nesse nível de atenção.

Diante do elevado fluxo de pacientes que necessitam de internação para tratamento da fase aguda de COVID-19, juntamente à capacidade limitada de leitos hospitalares, em diversos países, os pacientes recebem alta, independentemente do seu estado físico-funcional. (BELLI et al., 2020) O’Brien e demais autores (2020) identificaram que as necessidades por cuidados relacionados à saúde física, psicológica e cognitiva persistem após a resolução clínica da doença e que quase $50 \%$ dos pacientes necessitarão de acompanhamento especializado de médio a longo prazo. 
Em uma análise retrospectiva dos registros de pacientes avaliados na alta para casa, um terço dos pacientes evoluiram com baixa aptidão física e em $47,5 \%$ dos pacientes foi identificado comprometimento no desempenho das AVD. Esses resultados alertam para a demanda persistente por cuidados e reabilitação após a alta e a necessidade de equipes interdisciplinares em todos os níveis de atenção à saúde. (BELLI et al., 2020) Uma revisão sistemática dos resultados de epidemias de coronavírus anteriores destacou que $41 \%$ dos pacientes tiveram uma redução da capacidade aeróbia aos três meses pós-doença. (AHMAD; RATHORE, 2020) Problemas de tolerância ao exercício ou redução da capacidade das atividades diárias estão associados à fadiga (HALPIN et al., 2020), contribuindo para a redução do desempenho físico e capacidade para o trabalho.

Além das limitações para as AVD que garantem o autocuidado e a manutenção da vida prática, as repercussões da COVID-19 podem causar restrições na participação social em decorrência das disfunções físicas e mentais que persistem após a recuperação da doença. Entre os participantes de uma pesquisa na França, em que 56 indivíduos eram trabalhadores ativos antes de serem acometidos pela COVID-19, 38 (68\%) haviam retornado ao trabalho em um tempo médio de 111 dias após a hospitalização (GARRIGUES et al., 2020), e dentre 32 pacientes pós-UTI de uma coorte no Reino Unido, 22 (69\%) relataram novos problemas de mobilidade, autocuidado ou atividades usuais, entre 29 dias e 71 dias após alta hospitalar. (HALPIN et al., 2020)

Apesar da recomendação dos especialistas a favor da reabilitação após casos graves de infecção por COVID-19, vários fatores podem comprometer o encaminhamento ou a adesão à reabilitação, como a falta de orientação da equipe de saúde, a condição pré-infecção, que inclui sedentarismo e obesidade, a disponibilidade de serviços de reabilitação pulmonar, a dificuldade para 
o deslocamento e acesso ao centro de reabilitação e a situação financeira dos pacientes. Há uma forte reivindicação sobre a necessidade de triagem e encaminhamento de pacientes que foram hospitalizados, especialmente em UTI para a reabilitação. (POLASTRI et al., 2020) A oferta desse serviço, portanto, deve acontecer de forma acessível, atrativa, simples e direcionada, para maximizar a probabilidade de aceitação e recuperação desses indivíduos.

Compreende-se que a reabilitação após o COVID-19 deve ser semelhante àquela fornecida para muitos pacientes assistidos em unidades de reabilitação que foram afetados por outras doenças ou lesões. Alguns podem apresentar várias sequelas associadas à doença viral e à permanência prolongada na UTI, possivelmente incluindo ventilação mecânica. Muitos terão morbidades preexistentes agravadas ou descompensadas, requerendo cuidados especializados e prolongados. Uma avaliação completa e um plano de tratamento individualizado e progressivo com enfoque na recuperação da função, envolvimento em atividades e o retorno à participação na sociedade ajudarão os pacientes a maximizarem sua qualidade de vida.

Ao longo da pandemia, o fisioterapeuta tem sido reconhecido como profissional fundamental para a recuperação e alta hospitalar, contribuindo para a redução da mortalidade e sequelas causadas pelas manifestações fisiopatológicas da doença e pela internação prolongada. Desse modo, é fundamental que as futuras pesquisas investiguem os impactos da COVID-19 na limitação de atividade e na restrição de participação nos indivíduos que se recuperam da doença, a fim de oferecer embasamento científico para avaliação e desenvolvimento de modelos de cuidados, bem como ferramentas que contemplem as expectativas e necessidades de saúde frente aos novos desafios. No cenário pós-pandemia que já se desenha, esse profissional exercerá papel-chave nos níveis primário e secundário de atenção à saúde no resgate da funcionalidade e promoção da qualidade de vida das pessoas. 


\section{Desafios para a oferta de atendimento de fisioterapia na atenção primária à saúde e o enfrentamento da pandemia de COVID-19}

A pandemia de COVID-19 e suas sequelas imprimem ainda mais desafios, sobre os serviços de Fisioterapia, para o cumprimento de ações que envolvam promoção, prevenção e recuperação da população ao considerarmos os princípios de direito à saúde universal, equânime e integral. Cabe destacar que o Brasil instituiu desde 2012 uma Rede de Cuidados à Pessoa com Deficiência (RCPD), cujas ações de reabilitação em saúde devem ser transversais a diferentes pontos de cuidado, com vistas a garantir o acesso aos serviços desde a APS até a atenção hospitalar. (DUBOW; GARCIA; KRUG, 2018) Contudo, embora as necessidades de reabilitação em saúde tenham crescido, estudos são unânimes ao relatarem a fragilidade histórica da ampliação e organização de ações e serviços de reabilitação em saúde no Brasil (DUBOW; GARCIA; KRUG, 2018; CAETANO; SAMPAIO; COSTA, 2018; RODES et al., 2017) Para tanto, se apresenta como problema o modo como os sistemas de saúde garantirão o acesso ao cuidado dos usuários que se recuperaram dos efeitos agudos da COVID-19 e quais serão as demandas de reabilitação de médio e longo prazo.

A APS no Brasil possui papel estratégico para organização do SUS e deve contribuir para qualificar sua capacidade de resposta às necessidades sociais e de saúde vivenciadas pela população. À APS cabe organizar o escopo de suas práticas, a fim de garantir o primeiro contato dos usuários na rede de serviços, a longitudinalidade, a integralidade e a coordenação do cuidado. Somado a esses atributos, o processo de trabalho deve respeitar a orientação familiar, comunitária e a competência cultural. (BRASIL, 2017; STARFIELD, 2002) De acordo com o Ministério da Saúde (MS), até junho de 2020, 75\% da população brasileira era coberta 
pelas equipes do nível primário evidenciando, portanto, capilarização dos serviços por todo território nacional (BRASIL, 2020c) e revelando o potencial estratégico do país para o enfrentamento da pandemia da COVID-19. (SARTI et al., 2020) O nível primário tem sido apontado como o primeiro contato dos usuários com casos leves e moderados da COVID-19 (DUNLOP et al., 2020), bem como a identificação dos casos mais graves que devem ser encaminhados para os serviços especializados. (BRASIL, 2020b)

Na APS, destaca-se o papel estratégico do Núcleo Ampliado de Saúde da Família e Atenção Básica (NASF-AB), no qual há inserção do fisioterapeuta e cujo propósito visa expandir a oferta de ações, qualificar práticas e buscar a resolutividade do cuidado. (BRASIL, 2008, 2017) No contexto da pandemia, compete a esse profissional prevenir agravos, promover saúde e reabilitar os usuários com sequelas da COVID-19. Ressalta-se que as ações de prevenção e promoção não são específicas da categoria, pois compõem saberes e práticas do campo, compartilhadas entre os distintos membros das equipes da APS. (CAMPOS; DOMITTI, 2007) Nesse sentido, o MS elenca como possibilidades de atuação: estabelecer e divulgar orientações para a prevenção, o controle e a mitigação da transmissão da COVID-19 com informações sobre a doença, higiene das mãos, etiqueta respiratória e medidas de proteção individuais e coletivas com recomendação de isolamento domiciliar. (BRASIL, 2020a)

Do ponto de vista da reabilitação, é sabido que a lógica do NASF-AB, na qual o profissional da Fisioterapia atua, é singular em relação às práticas assistenciais operadas nos serviços de atenção especializada. O fisioterapeuta cumpre um papel fundamental para a produção do cuidado na APS, atuando no controle de risco, em intervenções coletivas para controle dos fatores que podem conduzir ao adoecimento, e também no controle de danos, delineado por intervenções individuais. (BISPO JÚNIOR, 2010) 
No contexto da pandemia, essa perspectiva deve ser mantida e qualificada para permear a busca pela recuperação dos distúrbios cinético-funcionais dos sujeitos acometidos pela COVID-19. É possível ainda, a realização do apoio matricial para demandas vinculadas ao movimento humano e articulação com os serviços da rede para pactuação de fluxos e monitoramento dos casos. (BARROS et al., 2015; BRASIL, 2014; OLIVEIRA et al., 2020; PORTELA; GRABOIS; TRAVASSOS, 2020)

Atenção especial deve ser empreendida no cuidado domiciliar para usuários restritos ao leito ou com comprometimento da funcionalidade. Nesse cenário, deve-se orientar os familiares, com atividades que possam ser realizadas, utilizando inclusive os objetos do cotidiano. A produção de materiais educativos impressos ou por mídias digitais revela-se com uma ferramenta potencialmente favorável às limitações impostas. Da mesma forma, o telessaúde e suas possibilidades contribuem para o acesso, a vinculação e a vigilância dos casos com comprometimento da funcionalidade decorrente da COVID-19. (ASSOBRAFIR, 2020; FALVEY; KRAFFT; KORNETTI, 2020; LOZANNO et al., 2020) Destaca-se, também, a importância dos serviços de base comunitária - domiciliar e ambulatorial -, já que a maioria das pessoas que recebe alta hospitalar pós-COVID-19 não terá acesso a serviços ambulatoriais especializados de fisioterapia oriundos de serviços públicos. (SMITH et al., 2020)

$\mathrm{Na}$ literatura internacional, foram identificados vários desafios para a oferta de atendimentos de fisioterapia na fase pós-hospitalar da COVID-19. Esses incluíam a escassez de profissionais fisioterapeutas nos serviços públicos e privados (LOZANNO et al., 2020), além de questões de saúde e segurança dos profissionais no trabalho (LOZANNO et al., 2020; SHEERY, 2020;) Ademais, foram também elencadas outras dificuldades, tais como o fechamento de serviços com redução do acesso ao tratamento (MINGHELLI et al., 2020; FALVEY; KRAFFT; KORNETTI, 2020), a necessidade de treinamento 
dos profissionais (SHEERY, 2020), mudanças organizacionais (AMATYA; KHAN, 2020; SIMONELLI et al., 2020) e a adoção de novas práticas de saúde, a exemplo da telereabilitação. (LOZANNO et al., 2020)

No Brasil, alguns desafios crônicos da oferta de atenção em Fisioterapia se apresentam ainda mais latentes no cenário da pandemia de COVID-19. Nos documentos normativos do SUS, as diretrizes que norteiam as atribuições dos fisioterapeutas nos NASF-AB ainda estão centradas em concepções que remetem direcionalidade das práticas de saúde que, de certa forma, privilegiam o componente da reabilitação física, secundarizando múltiplas potencialidades de atuação profissional na prevenção e na promoção da saúde.

Somado a isso, a mudança no financiamento da APS, mediante a Portaria $n^{\circ}$ 2.979/2019, que instituiu o Programa Previne Brasil, coloca em risco a manutenção dos núcleos de apoio, dada a não garantia de recursos para o NASF-AB, enquanto componente de ação estratégica da APS. Isso, consequentemente, ameaça o acesso da população às distintas categorias profissionais que compõem este arranjo organizacional, incluindo fisioterapeutas. (BRASIL, 2019; SETA; OCKÉ-REIS; RAMOS, 2020) Ao considerarmos a demanda reprimida pelas condições crônicas, além da necessidade de resposta ao novo quadro epidemiológico, imposto pela pandemia, não garantir aporte financeiro para custeio do NASF-AB enquanto ação estratégica pode resultar na desassistência de parte da população e impossibilitar a APS de atuar no enfrentamento da pandemia. (MEDINA et al., 2020)

Outros estudos identificaram entraves relacionados a precárias condições de trabalho reveladas por fragilização dos vínculos, falta de materiais para atividades técnico-pedagógicas, falta de insumos, transporte limitado ou mesmo ausente para deslocamento de fisioterapeutas em territórios remotos ou de difícil 
acesso. (BISPO JÚNIOR; MOREIRA, 2018) Somado a isso, podem ser também elencados a educação permanente insuficiente e inadequada (BISPO JÚNIOR; MOREIRA, 2017), a frágil retaguarda assistencial de reabilitação na rede locorregional para atender à demanda (NASCIMENTO et al., 2018), a necessidade de aprimorar o desenvolvimento da prática baseada em evidências (SCHNEIDER; PEREIRA; FERRAZ, 2018), a falta de informatização, de recursos tecnológicos e de capacitação das equipes sobre COVID-19, bem como a fragilidade na oferta dos equipamentos de proteção necessários. (BOUSQUAT et al., 2020)

Em vários cenários, a pandemia de COVID-19 gerou desafios relativos à necessidade de mudanças organizacionais nos serviços de saúde. Na Itália, por exemplo, as mudanças incluíram desde a redefinição de papéis entre os profissionais à dinâmica dos agendamentos nos serviços, incluindo turnos de trabalho e redefinição de protocolos de atendimentos fisioterapêuticos. (SIMONELLI et al., 2020) No campo da saúde e segurança do trabalho, estudos ratificam que a profissão de Fisioterapia é uma das mais vulneráveis à contaminação pela COVID-19. (LOZANNO et al., 2020; BOUSQUAT et al., 2020) Como alternativa, foram observadas mudanças nos meios e instrumentos de trabalho em reabilitação. Na Espanha, foi identificado o emprego de Tecnologias de Informação e Comunicação (TICs), com uso de telefone para monitoramento de pacientes, da prescrição de tratamentos digitalizada, da realização de vídeos explicativos, do tratamento por videoconferência síncrona e do teleatendimento em Fisioterapia. (LOZANNO et al., 2020)

A previsão de aumento na demanda por reabilitação significa que a capacidade de fornecer reabilitação precisa ser ampliada. Isso não será alcançado somente treinando mais especialistas ou desenvolvendo ferramentas tecnológicas distantes da realidade clínica e social da prática e dos usuários. Será necessária uma 
força de trabalho de reabilitação mais diversa para atender a esse desafio, usando a capacidade e habilidades de diferentes setores além das organizações de saúde. A telessaúde configura-se como uma grande oportunidade para os serviços de saúde e de assistência social transformarem a forma como oferecem a reabilitação, redesenhando as trajetórias do paciente entre o hospital e sua casa. (BIASE, 2020)

As ferramentas para a realização de ações de telessaúde compreendem softwares, plataformas digitais e aplicativos que possibilitam teleconsulta, telediagnóstico, telemonitoramento e telerreabilitação que são disponibilizados por serviços e profissionais de saúde de forma remota. Nesse contexto, esses recursos são utilizados principalmente em duas vertentes:

a. para diagnóstico, vigilância e monitoramento dos casos suspeitos, confirmados e recuperados de COVID-19 que necessitam de reabilitação após a fase aguda (DANTAS; BARRETO; FERREIRA, 2020; SIRE et al., 2020); e

b. para a manutenção e continuidade de tratamentos e intervenções que estavam em andamento previamente à pandemia. (BORG; STAM, 2020; NEGRINI et al., 2020)

Sendo assim, tais ferramentas têm exercido papel complementar, porém fundamental no enfrentamento da pandemia de COVID-19. (IANNACCONE et al., 2020) Pesquisadores têm se preocupado com outras condições de saúde que requerem reabilitação e sofreram o impacto indireto da pandemia interrompendo a reabilitação. Boldrini e demais autores (2020) destacaram a importância da adoção, sempre que possível, de modalidades alternativas para a oferta de atendimento. Eles apontaram que os serviços de reabilitação ambulatorial e domiciliar se organizaram para garantir o atendimento a pessoas com sequelas de diferentes origens, que se não tratados podem levar à incapacidade de longo 
prazo ou permanente. Os autores sugerem que as opções de teleatendimento devem ser consideradas para adiar os tratamentos presenciais ao mesmo tempo em que mantêm a relação terapêutica. (BOLDRINI et al., 2020)

Em geral, o uso da telessaúde tem apresentado bons resultados em desfechos clínico-funcionais, bem como tem atendido satisfatoriamente as expectativas dos pacientes diante do tratamento, sendo uma ferramenta importante a ser incorporada na prática clínica do fisioterapeuta. Entretanto, Dantas, Barreto e Ferreira (2020) alertam para as questões de segurança dos dados, da falta de regulamentação específica e sobre a necessidade de capacitar os profissionais e pacientes para o uso dessa tecnologia. Além disso, existe o risco de segregação e intensificação das desigualdades sociais devido ao acesso restrito aos equipamentos e rede de internet. Autores chamam a atenção para o fato de que o telessaúde pode não ser viável para todos os usuários como opção exclusiva ao considerarmos questões socioeconômicas ou necessidades especiais. (FALVEY; KRAFFT; KORNETTI, 2020) É fundamental que haja esforços no sentido de adaptar e verificar novos modos de oferecer reabilitação incluindo o uso de recursos on-line e digitais, entretanto, é preciso considerar que pessoas mais velhas tendem a ter menor alfabetização digital, perdas sensoriais e/ou deficiência cognitiva o que pode intensificar as desigualdades.

\section{Considerações finais}

O impacto no desempenho funcional decorrente das repercussões clínicas da infecção pelo SARS-CoV-2 traz um grande desafio para a oferta de atendimento de Fisioterapia. Observou-se que boa parte da produção científica era composta por textos opinativos, consenso de especialistas e orientações normativas baseadas em recomendações institucionais. Nesse sentido, torna-se necessário 
fomentar a condução de uma série de estudos mais robustos sobre o prognóstico e a sobrevida desses pacientes, além de pesquisas que avaliem o efeito das diferentes intervenções fisioterapêuticas sobre a funcionalidade. Ademais, também carecem de maior análise as questões voltadas para a pesquisa no campo do planejamento e gestão de serviços de reabilitação para COVID-19, incluindo estudos sobre as demandas por serviços de fisioterapia que considerem a necessária ampliação da oferta no SUS.

É inegável que a pandemia de COVID-19 modificou a forma como vivemos, cuidamos e trabalhamos, e o processo de reabilitação deve se adaptar a uma nova organização e gestão de cuidado e assistência à saúde. Mesmo quando a reabilitação presencial for possível, é provável que parte dessa assistência possa acontecer de modo remoto para ampliar o acesso aos recursos fisioterapêuticos com maior frequência, otimizando os ganhos funcionais. Como perspectiva, apresenta-se a possibilidade de orientar os serviços de Fisioterapia em práticas de atenção inovadoras capazes de responder às necessidades de saúde da população.

\section{Referências}

AHMAD, I.; RATHORE, F. A. Neurological manifestations and complications of COVID-19: A literature review. Journal of Clinical Neuroscience, Melbourne, v. 77, p. 8-12, 2020. DOI: https://doi.org/10.1016/j.jocn.2020.05.017. Disponível em: https://www.ncbi.nlm.nih.gov/pmc/articles/PMC7200361/pdf/main.pdf. Acesso em: 3 set. 2020.

ALHAZZANI, W. et al. Surviving Sepsis Campaign: guidelines on the management of critically ill adults with Coronavirus Disease 2019 (COVID-19). Intensive Care Medicine, New York, v. 46, n. 5, p. 854-887, 2020. DOI: 10.1007/ s00134-020-06022-5. Disponível em: https://www.ncbi.nlm.nih.gov/pmc/articles/ PMC7101866/pdf/134_2020_Article_6022.pdf. Acesso em: 29 ago. 2020. 
AMATYA, B.; KHAN, F. Rehabilitation Response in Pandemics. American Journal of Physical Medicine \& Rehabilitation, Baltimore,v. 99, n. 8, p. 663-668. 2020.

APPLETON, R. T.; KINSELLA, J.; QUASIM, T. The incidence of intensive care unitacquired weakness syndromes: A systematic review. Journal of the Intensive Care Society, London, v. 16, n. 2, p. 126-136, 2015.

ASADI-POOYA, A. A.; SIMANI, L. Central nervous system manifestations of COVID19: A systematic review. Journal of the Neurological Sciences, Amsterdam, v. 413, p. 116832, 2020. DOI: 10.1016/j.jns.2020.116832. Disponível em: https://www.ncbi. nlm.nih.gov/pmc/articles/PMC7151535/pdf/main.pdf. Acesso em: 3 set. 2020.

ASSOCIAÇÃO BRASILEIRA DE FISIOTERAPIA RESPIRATÓRIA - ASSOBRAFIR. Recomendações para a atuação dos fisioterapeutas no âmbito da atenção primária à saúde (APS) de pacientes suspeitos ou diagnosticados com COVID-19. [São Paulo], 2020.

BARROS, J. O. et al. Estratégia do apoio matricial: a experiência de duas equipes do Núcleo de a poio à saúde da família (NASF) da cidade de São Paulo, Brasil. Ciência \& Saúde Coletiva, Rio de Janeiro, v. 20, n. 9, p. 2847-2856, 2015.

BATTAGLINI, D. et al. Chest physiotherapy: An important adjuvant in critically ill mechanically ventilated patients with COVID-19. Respiratory Physiology \& Neurobiology, Amsterdam, v. 282, p. 103529, 2020. DOI: 10.1016/j. resp.2020.103529. Disponível em: https://www.sciencedirect.com/science/article/ pii/S1569904820301877. Acesso em: 8 set. 2020.

BELLI, S. et al. Low physical functioning and impaired performance of activities of daily life in COVID-19 patients who survived the hospitalisation. The European Respiratory Journal, Sheffield, v. 56, n. 4, 2020. DOI: https://doi. org/10.1183/13993003.02096-2020. Disponível em: https://www.ncbi.nlm.nih.gov/ pmc/articles/PMC7411272/pdf/ERJ-02096-2020.pdf. Acesso em: 3 set. 2020.

BERGER, J. R. COVID-19 and the nervous system. Journal of Neurovirology, Houndmills, v. 26, n. 2, p. 143-148, 2020. https://doi.org/10.1007/ s13365-020-00840-5. Disponível em: https://www.ncbi.nlm.nih.gov/pmc/articles/ PMC7245181/pdf/13365_2020_Article_840.pdf. Acesso em: 7 set. 2020.

BIASE, S. et al. The COVID-19 rehabilitation pandemic. Age and Ageing, Oxford, v. 49, n. 5, p. 696-700, 2020. DOI: https://doi.org/10.1093/ageing/afaa118. Disponível em: https://www.ncbi.nlm.nih.gov/pmc/articles/PMC7314277/pdf/ afaa118.pdf. Acesso em: 25 ago. 2020. 
BISPO JÚNIOR, J. P. Fisioterapia e saúde coletiva: desafios e novas responsabilidades profissionais. Ciência \& Saúde Coletiva, Rio de Janeiro, v. 15, supl. 1, p. 1627-36, 2010.

BISPO JÚNIOR, J. P.; MOREIRA, D. C. Educação permanente e apoio matricial: formação, vivências e práticas dos profissionais dos Núcleos de Apoio à Saúde da Família e das equipes apoiadas. Cadernos de Saúde Pública, Rio de Janeiro, v. 33, n. 9, 2017.

BISPO JÚNIOR, J. P.; MOREIRA, D. C. Núcleos de apoio à saúde da família: concepções, implicações e desafios para o apoio matricial. Trabalho, Educação e Saúde, Rio de Janeiro, v. 16 n. 2, p. 683-702, maio/ago. 2018.

BOLDRINI, P. et al. First impact of COVID-19 on services and their preparation "Instant paper from the field" on rehabilitation answers to the COVID-19 emergency. European Journal of Physical and Rehabilitation Medicine, Torino, v. 56, n. 3, p. 319-322, 2020. DOI: 10.23736/S1973-9087.20.06303-0. Disponível em: https:// www.minervamedica.it/en/getfreepdf/T3VvKzVtZzFzUnpDU3MwMlo2TGF6a TgxZCtPVGpwVjNZcTBFakF1UXZvSjZleG5ya242RVpNQTk1Q0MwUTFqZw\%253D \%253D/R33Y2020N03A0319.pdf. Acesso em: 4 set. 2020.

BOLES, J. M. et al. Weaning from mechanical ventilation. The European Respiratory Journal, Sheffield, v. 29 n. 5, p. 1033-1056, 2007. DOI: https://doi. org/10.1183/09031936.00010206. Disponível em: https://erj.ersjournals.com/ content/erj/29/5/1033.full.pdf. Acesso em: 8 set. 2020

BORG, K.; STAM, H. Covid-19 and Physical and Rehabilitation Medicine. Journal of Rehabilitation Medicine. Uppsala, SWE, v. 52, n. 4, 2020.

DOI: 10.2340/16501977-2679. Disponível em: https://www.medicaljournals.se/jrm/ content/html/10.2340/16501977-2679. Acesso em: 5 set. 2020.

BOUSQUAT, A. et al. (coord.). Desafios da Atenção Básica no enfrentamento da pandemia da COVID-19 no SUS. Relatório de Pesquisa. Rio de Janeiro: Rede de Pesquisa em APS Abrasco, ago. 2020. Disponível em: https://redeaps.org.br/ wp-content/uploads/2020/08/Relato\%CC\%81 rioDesafiosABCovid19SUS.pdf. Acesso em: 15 set. 2020.

BRASIL. Ministério da Saúde. Portaria GM n 154, de 24 de janeiro de 2008. Cria os Núcleos de Apoio à Saúde da Família - NASF. Diário Oficial da União: seção 1, Brasília, DF, p. 38-42, mar. 2008. 
BRASIL. Ministério da Saúde. Portaria $n^{\circ} 1.565$, de 18 de junho de 2020. Estabelece orientações gerais visando à prevenção, ao controle e à mitigação da transmissão da COVID-19, e à promoção da saúde física e mental da população brasileira, de forma a contribuir com as ações para a retomada segura das atividades e o convívio social seguro. Diário Oficial da União: seção 1, Brasília, DF, n. 116, p. 64, 19 jun. 2020a.

BRASIL. Ministério da Saúde. Portaria n².436, de 21 de setembro de 2017. Aprova a Política Nacional de Atenção Básica, estabelecendo a revisão de diretrizes para a organização da Atenção Básica, no âmbito do Sistema Único de Saúde (SUS). Diário Oficial da União: seção 1, Brasília, DF, n. 183, p. 68, 22 set. 2017.

BRASIL. Ministério da Saúde. Portaria n².979, de 12 de novembro de 2019. Institui o Programa Previne Brasil, que estabelece novo modelo de financiamento de custeio da Atenção Primária à Saúde no âmbito do Sistema Único de Saúde, por meio da alteração da Portaria de Consolidação n 6/GM/MS, de 28 de setembro de 2017. Diário Oficial da União: seção 1, Brasília, DF, n. 220, p. 97, 13 nov. 2019.

BRASIL. Ministério da Saúde. Secretaria de Atenção Primária à Saúde. Protocolo do Manejo Clínico do Coronavírus (COVID-19) na Atenção Primária à Saúde (APS). Brasília, DF, 2020b. Disponível em: http://portalarquivos2.saude.gov.br/images/ pdf/2020/marco/24/20200323-ProtocoloManejo-ver05.pdf. Acesso em: 7 set. 2020.

BRASIL. Ministério da Saúde. Secretaria de Atenção à Saúde. Departamento de Atenção Básica. Núcleo de Apoio à Saúde da Família. Brasília, DF: Ministério da Saúde, 2014. (Cadernos de Atenção Básica, n. 39).

BRASIL. Ministério Da Saúde. Secretaria de Atenção Primária à Saúde.

Departamento de Saúde da Família. Histórico de cobertura da saúde da atenção básica. Brasília, DF: Ministério da Saúde 2020c. Disponível em: https://egestorab. saude.gov.br/paginas/acessoPublico/relatorios/relHistoricoCoberturaAB. xhtml;jsessionid=I9Mt+R4qOoNjcTPalSErRzF5. Acesso em: 7 set. 2020.

CAETANO, L. A.; SAMPAIO, R. F., COSTA, L. A. A expansão dos serviços de reabilitação no SUS à luz do arcabouço normativo federal. Revista de Terapia Ocupacional da Universidade de São Paulo, São Paulo, v. 29, n. 3, p. 195-203, set./dez. 2018.

CAMPOS, G. W. S.; DOMITTI, A. C. Apoio matricial e equipe de referência: uma metodologia para gestão do trabalho interdisciplinar em saúde. Cadernos de Saúde Pública, Rio de Janeiro, v. 23, n. 2, p. 399-407, fev. 2007. 
CHERRY, J. D.; KROGSTAD, P. SARS: the first pandemic of the 21 st century. Pediatric Research, Basel, v. 56, n. 1, p. 1-5, July 2004. DOI: 10.1203/01. PDR.0000129184.87042.FC. Disponível em: https://www.ncbi.nlm.nih.gov/pmc/ articles/PMC3329048/09. Acesso em: 9 set. 2020.

CIPOLLARO, L. et al. Musculoskeletal symptoms in SARS-CoV-2 (COVID-19) patients. Journal of Orthopaedic Surgery and Research, London, v. 15, n. 178, p. 1-7, 2020.

DANTAS, L. O; BARRETO, R. P. G.; FERREIRA, C. H. J. Digital physical therapy in the COVID-19 pandemic. Brazilian Journal of Physical Therapy, São Carlos, v. 24, n. 5, p. 381-383, 2020. DOI: https://doi.org/10.1016/j.bjpt.2020.04.006. Disponível em: http://www.rbf-bjpt.org.br/en-pdf-S1413355520304020. Acesso em: 7 set. 2020.

DEAN, E. et al. Translating COVID-19 Evidence to Maximize Physical Therapists' Impact and Public Health Response. Physical Therapy, Alexandria, v. 100, n. 9, p. 1458-1464. DOI: doi.org/ 10.1093/ptj/pzaa115. Disponível em: https://academic. oup.com/ptj/article/100/9/1458/5862054. Acesso em: 8 set. 2020.

DING, Y. et al. The clinical pathology of severe acute respiratory syndrome (SARS): a report from China. The Journal of Pathology, London, v. 200, n. 3, p. 282-289, July 2003.

DOCHERTY, A. B. et al. Features of 20133 UK patients in hospital with COVID-19 using the ISARIC WHO Clinical Characterisation Protocol: prospective observational cohort study BMJ, London, v. 369, p. m1985, 2020. DOI: https://doi.org/10.1136/bmj.m1985. Disponível em: https://www.bmj.com/ content/bmj/369/bmj.m1985.full.pdf. Acesso em: 8 set. 2020.

DUBOW, C.; GARCIA, E. L.; KRUG, S. B. F. Percepções sobre a Rede de Cuidados à Pessoa com Deficiência em uma Região de Saúde. Saúde Debate, Rio de Janeiro, v. 42, n. 117, p. 455-467, abr./jun. 2018.

DUNLOP, C. et al. The coronavirus outbreak: the central role of primary care in emergency preparedness and response. BJGP Open, London, v. 4, n. 1, 2020. DOI: https:// doi.org/10.3399/bjgpopen20X101041. Disponível em: https://bjgpopen.org/content/bjgpoa/4/1/bjgpopen20X101041.full.pdf. Acesso em: 29 ago. 2020.

FALVEY, J. R.; KRAFFT, C.; KORNETTI, D. The Essential Role of Homeand Community-Based Physical Therapists During the COVID-19 Pandemic. Physical Therapy, Alexandria, VA, v. 100, n. 7, p. 1058-1061, 2020. 
FAN, E. et al. An Official American Thoracic Society/European Society of Intensive Care Medicine/Society of Critical Care Medicine Clinical Practice Guideline: Mechanical Ventilation in Adult Patients with Acute Respiratory Distress Syndrome. American Journal of Respiratory and Critical Care Medicine, New York, v. 195, n. 9, p. 1253-1263, 2017. DOI: 10.1164/rccm.201703-0548ST. Disponível em: https://www.atsjournals.org/doi/pdf/10.1164/rccm.201703-0548ST. Acesso em: 25 ago. 2020.

FANG, D. SARS: facts and considerations of the orthopaedic community. Journal of Orthopaedic Surgery, Hong Kong, v. 1, n. 1, p. 3-5, June 2003.

FERINI-STRAMBI, L.; SALSONE, M. COVID-19 and neurological disorders: are neurodegenerative or neuroimmunological diseases more vulnerable? Journal of Neurology, New York, 2020. DOI: https://doi.org/10.1007/s00415-020-10070-8, Disponível em: https://www.ncbi.nlm.nih.gov/pmc/articles/PMC7372546/ pdf/415_2020_Article_10070.pdf. Acesso em: 6 set. 2020.

FRIJA-MASSON, J. et al. Functional characteristics of patients with SARS-CoV-2 pneumonia at 30 days post infection. The European Respiratory Journal, Copenhagen, v. 56, n. 2, p. 2001754, 2020. https://doi. org/10.1183/13993003.01754-2020. Disponível em: https://www.ncbi.nlm.nih.gov/ pmc/articles/PMC7301832/pdf/ERJ-01754-2020.pdf. Acesso em: 9 set. 2020.

GARRIGUES, E. et al. Post-discharge persistent symptoms and health-related quality of life after hospitalization for COVID-19. The Journal of Infection, London, 2020. DOI:10.1016/j.jinf.2020.08.029. Disponível em: https://www.ncbi.nlm.nih.gov/pmc/ articles/PMC7445491/pdf/main.pdf. Acesso em: 8 set. 2020.

GHANNAM, M. et al. Neurological involvement of coronavirus disease 2019: a systematic review. Journal of Neurology, Berlin, 2020. DOI: https://doi. org/10.1007/s00415-020-09990-2. Disponível em: https://www.ncbi.nlm.nih.gov/ pmc/articles/PMC7304377/. Acesso em: 5 set. 2020.

GRIFFITH, James. Musculoskeletal Complications of Severe Acute Respiratory Syndrome. Seminars in Musculoskeletal Radiology, New York, v. 15, n. 5, p. 554-560, 2011. DOI: 10.1055/s-0031-1293500. Disponível em: https://www.thieme-connect. com/products/ejournals/abstract/10.1055/s-0031-1293500. Acesso em: 5 set. 2020.

GUÉRIN, C. et al. Prone positioning in severe acute respiratory distress syndrome. The New England Journal of Medicine, Boston, v. 368, n. 23, p. 2159-2168, 2013. 
HALPIN, S. J. et al. Postdischarge symptoms and rehabilitation needs insurvivors of COVI-19 infection: A crossectional evaluation. Journal of Medical Virology, New York, 2020. DOI: 10.1002/jmv.26368. Disponível em: https://onlinelibrary.wiley. com/doi/epdf/10.1002/jmv.26368. Acesso em: 29 ago. 2020.

HERRIDGE, M. S. et al. Functional disability 5 years after acute respiratory distress syndrome. The New England Journal of Medicine, Boston, v. 364, n. 14, p. 1293-1304, 2011. DOI: 10.1056/NEJMoa1011802. Disponível em: https://www.nejm.org/doi/pdf/10.1056/NEJMoa1011802?articleTools=true. Acesso em: 3 set. 2020.

HEYDARI, K. et al. Clinical and paraclinical characteristics of COVID-19 patients: a systematic review and meta-analysis. MedRxiv, [s. I.], 2020. Disponível em: https://www.medrxiv.org/content/10.1101/2020.03.26.20044057v. Acesso em: 27 abr. 2020.

HODGSON, C. L. et al. Expert consensus and recommendations on safety criteria for active mobilization of mechanically ventilated critically ill adults. Critical Care, London, v. 18, n. 6, p. 658, 2014. DOI: 10.1186/s13054-014-0658-y. Disponível em: https://ccforum.biomedcentral.com/articles/10.1186/s13054-014-0658-y. Acesso em: 2 set. 2020.

HUSAIN, R. et al. Rhabdomyolysis as a manifestation of a severe case of COVID19: A case report. Radiology Case Reports, Seattle, v. 15, n. 9, p. 1633-1637, 2020. DOI: 10.1016/j.radcr.2020.07.003. Disponível em: https://www.ncbi.nlm.nih.gov/ pmc/articles/PMC7340044/pdf/main.pdf. Acesso em: 5 set. 2020.

IANNACCONE, S. et al. Role of Rehabilitation Department for Adult Individuals With COVID-19: The Experience of the San Raffaele Hospital of Milan. Archives of Physical Medicine and Rehabilitation, Philadelphia, v. 101, n. 9, p. 1656-1661, 2020. DOI: https://doi.org/10.1016/j.apmr.2020.05.015. Disponível em: https://www. archives-pmr.org/article/S0003-9993(20)30365-8/fulltext. Acesso em: 7 set. 2020

KALIRATHINAM, D.; GURUCHANDRAN, R.; SUBRAMANI, P. Comprehensive physiotherapy management in covid-19 - a narrative review. Scientia Medica, Porto Alegre, v. 30, n. 1, p. 38030, 2020. DOI: 10.15448/1980-6108.2020.1.38030. Disponível em: https://revistaseletronicas.pucrs.br/ojs/index.php/scientiamedica/ article/view/38030/19715. Acesso em: 22 ago. 2020.

LAU, H. M. C. et al. The impact of sever acute respiratory syndrome on the physical profile and quality of life. Archives of Physical Medicine and Rehabilitation, Philadelphia, v. 86, n. 6, 1134-1140, June 2005. 
LAZZERI, M. et al. Respiratory physiotherapy in patients with COVID-19 infection in acute setting: a Position Paper of the Italian Association of Respiratory Physiotherapists (ARIR). Monaldi Archives for Chest Disease, Pavia, v. 90, n. 1, 2020. DOI:10.4081/monaldi.2020.1285. Disponível em: https://www.monaldi-archives. org/index.php/macd/article/view/1285/1003 Acesso em: 1 set. 2020.

LECHIEN, J. R. et al. Olfactory and gustatory dysfunctions as a clinical presentation of mild-to-moderate forms of the coronavirus disease (COVID-19): a multicenter European study. European Archives of Oto-rhino-laryngology, Heidelberg, v. 277, n. 8, p. 2251-2261, 2020. DOI: 10.1007/s00405-020-05965-1. Disponível em: https://www.ncbi.nlm.nih.gov/pmc/articles/PMC7134551/pdf/405_2020_ Article_5965.pdf. Acesso em: 5 set. 2020.

LEONARDI, M.; PADOVANI, A.; MCARTHUR, J. C. Neurological manifestations associated with COVID-19: a review and a call for action. Journal of Neurology, New York, v. 267, n. 6, p. 1573-1576, 2020. DOI: https://doi. org/10.1007/s00415-020-09896-z. Disponível em: https://www.ncbi.nlm.nih.gov/ pmc/articles/PMC7238392/pdf/415_2020_Article_9896.pdf. Acesso em: 7 set. 2020.

LOZANNO, R. R. et al. La fisioterapia española en tiempos de pandemia de la COVID-19. Fisioterapia, Madrid, v. 42, n. 4, p. 224-225, luglio/agosto 2020.

MADJID, M et al. Potential Effects of Coronaviruses on the Cardiovascular System: A Review. JAMA Cardiology. Chicago, v. 5, n. 7, p. 831-840, 2020. DOI: 10.1001/ jamacardio.2020.1286. Disponível em: https://jamanetwork.com/journals/ jamacardiology/fullarticle/10.1001/jamacardio.2020.1286. Acesso em: 7 set. 2020.

MEDINA, M. G. et al. Atenção primária à saúde em tempos de COVID-19: o que fazer? Cadernos de Saúde Pública, Rio de Janeiro, v. 36, n. 8, jun. 2020. Disponível em: http://cadernos.ensp.fiocruz.br/csp/artigo/1140/atencao-primariaa-saude-em-tempos-de-COVID-19-o-que-fazer. Acesso em: 29 ago. 2020.

MINGHELLI, B. et al. Physiotherapy services in the face of a pandemic. Revista da Associação Médica Brasileira, São Paulo, v. 66, n. 4, p. 491-497, abr. 2020.

MONTALVAN, V. et al. Neurological manifestations of COVID-19 and other coronavirus infections: A systematic review. Clinical Neurology and Neurosurgery, Amsterdam, v. 194, July 2020. 105921 DOI: https://doi.org/10.1016/j. clineuro.2020.105921. Disponível em: https://www.sciencedirect.com/science/ article/abs/pii/S030384672030264X?via\%3Dihub. Acesso em: 20 ago. 2020. 
NASCIMENTO, C. M. B. et al. Configurações do processo de trabalho em núcleos de apoio à saúde da família e o cuidado integral. Trabalho, Educação e Saúde, Rio de Janeiro, v. 16, n. 3, p. 1135-1156, dez. 2018.

NEEDHAM, D. M. et al. Core Outcome Measures for Clinical Research in Acute Respiratory Failure Survivors. An International Modified Delphi Consensus Study. American Journal of Respiratory and Critical Care Medicine, New York, v. 196, n. 9, p. 1122-1130, 2017.

NEGRINI, S. et al. Telemedicine from research to practice during the pandemic "Instant paper from the field" on rehabilitation answers to the COVID-19 emergency. European Journal of Physical and Rehabilitation Medicine, Torino, v. 56 n. 3, p. 327-30, 2020. DOI: 10.23736/S1973-9087.20.06331-5. Disponível em: https://www.minervamedica.it/en/getfreepdf/aW9TUWJLNIhqTDdkY1IVakJodz ZTMUo3ZEE1cWFVSWN1TE1CbmtSRnFIZ1Q0ak5kSXZISEXJMXIEeWtvSi93MQ \%253D\%253D/R33Y2020N03A0327.pdf. Acesso em: 29 ago. 2020.

O'BRIEN, H. et al. An integrated multidisciplinary model of COVID-19 recovery care. Irish Journal of Medical Science, [s. I.], 2020. DOI: https://doi. org/10.1007/s11845-020-02354-9. Disponível em: https://link.springer.com/ article/10.1007\%2Fs11845-020-02354-9. Acesso em: 25 ago. 2020.

OLIVEIRA, E. T. de et al. A prática do núcleo de apoio à saúde da família do Recife no enfrentamento à pandemia COVID-19. APS em Revista, Belo Horizonte, v. 2, n. 2, p. 142-150, 9 jun. 2020.

PINZON, R.T. et al. Neurologic Characteristics in Coronavirus Disease 2019 (COVID-19): A Systematic Review and Meta-Analysis. Frontiers in Neurology, Lausanne, v. 11, p. 565, 2020. DOI: 10.3389/fneur.2020.00565. Disponível em: https://www.ncbi.nlm.nih.gov/pmc/articles/PMC7273516/pdf/fneur-11-00565.pdf. Acesso em: 5 set. 2020.

POLASTRI, M. et al. COVID-19 and pulmonary rehabilitation: preparing for phase three. The European Respiratory Journal, Copenhagen, v. 55, n. 6, 2020. DOI: https:// doi.org/10.1183/13993003.01822-2020. Disponível em: https://erj.ersjournals.com/ content/55/6/2001822. Acesso em: 7 set. 2020.

PORTELA, M. C.; GRABOIS, V.; TRAVASSOS, C. Matriz linha de cuidado COVID-19 na rede de atenção à saúde. Observatório COVID-19, [Rio de Janeiro], 2020. 
RIGHETTI, R. F. et al. Physiotherapy Care of Patients with Coronavirus Disease 2019 (COVID-19) - A Brazilian Experience. Clinics, São Paulo, v. 75, 2020. DOI: 10.6061/ clinics/2020/e2017. Disponível em: https://www.clinicsjournal.com/wp-content/ uploads/articles_xml/1807-5932-clin-75-e2017/1807-5932-clin-75-e2017.pdf. Acesso em: 20 ago. 2020.

RODES, C. H. et al. O acesso e o fazer da reabilitação na Atenção Primária à Saúde. Fisioterapia e Pesquisa, São Paulo, v. 24, n. 1, p. 74-82. 2017.

SARTI, T.D. et al. Qual o papel da Atenção Primária à Saúde diante da pandemia provocada pela COVID-19? Epidemiologia e Serviços de Saúde, Brasília, DF, v. 29, n. 2, p. e2020166, 2020.

SCHMIDT, G. A. et al. Liberation From Mechanical Ventilation in Critically III Adults: Executive Summary of an Official American College of Chest Physicians/ American Thoracic Society Clinical Practice Guideline. Chest, New York, v. 151, n. 1, p. 160-165, 2017. DOI: https://doi.org/10.1016/j.chest.2016.10.037. Disponível em: https://journal.chestnet.org/action/showPdf?pii=S0012-3692\%2816\%2962325-5. Acesso em: 3 set. 2020.

SCHNEIDER, L. R.; PEREIRA, R. P. G.; FERRAZ, L. A prática baseada em evidência no contexto da Atenção Primária à Saúde. Saúde Debate, Rio de Janeiro, v. 42, n. 118, p. 594-605, set. 2018.

SERRANO-CASTRO, P. J. et al. Influencia de la infección SARS-CoV-2 sobre enfermedades neurodegenerativasy neuropsiquiátricas: ¿una pandemia demorada? Neurología, Barcelona, v. 35, n. 4, p. 245-251, 2020. DOI: https://doi. org/10.1016/j.nrl.2020.04.002. Disponível em: https://www.ncbi.nlm.nih.gov/pmc/ articles/PMC7164900/pdf/main.pdf. Acesso em: 27 ago. 2020.

SETA, M. H.; OCKÉ-REIS, C. O.; RAMOS, A. L. P. Programa Previne Brasil: O ápice das ameaças à Atenção Primária à Saúde? Ciência \& Saúde Coletiva, Rio de Janeiro, mar. 2020. Disponível em: http://www.cienciaesaudecoletiva.com.br/artigos/programaprevine-brasil-o-apice-das-ameacas-a-atencao-primaria-a-saude/17535?id=17535. Acesso em: 23 ago. 2020.

SHEERY, L. M. Considerations for Postacute Rehabilitation for Survivors of COVID19. JMIR Public Health Surveill, Toronto, v. 6, n. 2, p. 19462, 2020.

SHI, Y. et al. An overview of COVID-19. Journal of Zhejiang University-Science B, Hangzhou, v. 21, p. 343-360, 2020. DOI: https://doi.org/10.1631/jzus.B2000083. Disponível em: https://link.springer.com/article/10.1631/jzus.B2000083. Acesso em: 27 ago. 2020. 
SIMONELLI, C. et al. How the COVID-19 infection tsunami revolutionized the work of respiratory physiotherapists: an experience from Northern Italy. Monaldi Archives for Chest Disease, Pavia, v. 90, n. 2, May 2020.

SIRE, A. et al. Systematic rapid living review on rehabilitation needs due to COVID-19: update as of April 30th, 2020. European Journal of Physical and Rehabilitation Medicine, Torino, v. 56, n. 3, p. 354-360, 2020. DOI: 10.23736/ S1973-9087.20.06378-9. Disponível em: https://www.minervamedica.it/en/ getfreepdf/Nkw1VVkxWIBGcHQ1NjZuWjJWTG5OSUZyTkgxRzlycFZWT21VM3V MaVJ5dGJSajBtZIJpYmIRNGVWdWVFaHIGaA\%253D\%253D/R33Y2020N03A0354. pdf Acesso em: 5 set. 2020.

SMITH, J. M. et al. Home and Community-Based Physical Therapist Management of Adults With Post-Intensive Care Syndrome. Physical Therapy, New York, v. 100, n. 7, p. 1062-1073, 2020.

STAM, H. J.; STUCKI, G.; BICKENBACH, J. COVID-19 and Post Intensive Care Syndrome: A Call for Action. Journal of Rehabilitation Medicine, Stockholm, v. 52, n. $4,2020$.

STARFIELD, B. Atenção primária: equilíbrio entre necessidades de saúde, serviços e tecnologia. Brasília, DF: UNESCO: Ministério da Saúde, 2002.

STAWICKI, S. P. et al. The 2019-2020 Novel Coronavirus (Severe Acute Respiratory Syndrome Coronavirus 2) Pandemic: A Joint American College of Academic International Medicine-World Academic Council of Emergency Medicine Multidisciplinary COVID-19 Working Group Consensus Paper. Journal of Global Infectious Diseases, Mumbai, v. 12, n. 2, p. 47-93, 2020. DOI: 10.4103/jgid. jgid_86_20. PMID: 32773996. Disponível em: https://www.ncbi.nlm.nih.gov/pmc/ articles/PMC7384689/. Acesso em: 15 set. 2020.

TANSEY, C. M. et al. One-Year Outcomes and Health Care Utilization in Survivors of Severe Acute Respiratory Syndrome. Archives of Internal Medicine, Chicago, v. 167, n. 12, p. 1312-1320, 2007. DOI: 10.1001/archinte.167.12.1312. Disponível em: https://jamanetwork.com/journals/jamainternalmedicine/fullarticle/10.1001/ archinte.167.12.1312. Acesso em: 7 set. 2020.

TURCOTTE, J. J. et al. Risk factors for severe illness in hospitalized Covid-19 patients at a regional hospital. PLoS One, California, v. 15, n. 8, p. e0237558, 2020. DOI: 10.1371/journal.pone.0237558. Disponível em: https://journals.plos. org/plosone/article/file?id=10.1371/journal. pone.0237558\&type=printable. Acesso em: 20 ago. 2020. 
WANG, L. et al. Clinical manifestations and evidence of neurological involvement in 2019 novel coronavirus SARS-CoV-2: a systematic review and meta-analysis. Journal of Neurology, New York, v. 267, n. 10, p. 2777-2789, 2020. DOI: https://doi.org/10.1007/s00415-020-09974-2. Disponível em: https:// www.ncbi.nlm.nih.gov/pmc/articles/PMC7288253/pdf/415_2020_Article_9974.pdf. Acesso em: 2 set. 2020.

WHITTAKER. A.; ANSON, M.; HARKY, A. Neurological Manifestations of COVID19: A systematic review and current update. Acta Neurologica Scandinavica, Copenhagen, v. 142, n. 1, p. 14-22, 2020. DOI: 10.1111/ane.13266.

Disponível em: https://onlinelibrary.wiley.com/doi/epdf/10.1111/ane.13266.

Acesso em: 19 ago. 2020.

WU, Z.; MCGOOGAN, J. M. Characteristics of and Important Lessons From the Coronavirus Disease 2019 (COVID-19) Outbreak in China: Summary of a Report of 72314 Cases From the Chinese Center for Disease Control and Prevention. JAMA, Chicago, v. 323, n. 13, p. 1239-1242, 2020. DOI:10.1001/jama.2020.2648.

Disponível em: https://jamanetwork.com/journals/jama/fullarticle/10.1001/ jama.2020.2648?alert=article\#. Acesso em: 6 set. 2020. 